\title{
Normal and Abnormal Colour Vision*
}

\author{
By Prof. H. E. Roaf
}

$\mathrm{R}^{\mathrm{H}}$

ECENTLY attention has been directed to the number of accidents caused by mechanically propelled vehicles. The use of coloured signals may lead to difficulties for drivers with defective colour vision. Until the colour vision of persons who seem to disregard the coloured lights is tested, we do not know to what extent coloured lights constitute a difficulty to motor drivers with defective colour vision. In any case the remedy is simple, as a difference in shape of the coloured lights would be sufficient to prevent mistakes. It is true that the relative positions of the lights and other data may help in the recognition of the colour, so that the problem is not so serious as in the case of railway and marine services.

The aim of this address is to discuss three aspects of the physiology of colour vision. The first aspect is the validity of the trichromatic hypothesis. There may not be many new things to be said, but a restatement of the arguments is useful as showing to what extent the hypothesis can be relied upon. The second aspect is the nature of the departures from normal colour vision of those with defective colour vision. The third aspect is a brief consideration of some theoretical views on the nature of colour-perceiving mechanisms.

\section{Physiological Meaning of Colodr}

Colours are visible in the spectrum, and we can recognise certain colours which seem unitary and distinct from all others, namely, red, yellow, green and blue. There are, however, other unitary sensations which must be considered, namely, white and black: these cannot be produced by stimulation with any one region of the spectrum. These two sensations are sometimes described as belonging to the colourless sensations, but psychoIogically one cannot separate them from a discussion on colour.

Thus we find that certain colours are related to definite regions of the spectrum, but there are other sensations which do not correspond to any single group of wave-lengths: the latter are the purples, white and black. All colours can be represented by fusion of lights from several regions of the spectrum, and the minimum number of regions is three. This physical relation is generally considered of paramount importance in the discussion of colour vision.

In 1802, Young postulated that there were

* From the presidential address before Section I (Physiology) of the British Association, delivered at Aberdeen on September 10. three sensory mechanisms, because all colours could be reproduced by a combination of three regions of the spectrum. There has always seemed some difficulty in reconciling this view with the psychological point of view that there are six distinct kinds of visual colour sensation, namely, red, yellow, green, blue, white and black. In the discussion of this problem, some of these simple psychological effects can be shown to be built up from other sensory processes. The discussion of the sensation of yellow occupies an important place, but before we deal with the sensation of yellow it is simpler to consider the sensations of white and of black.

Sensation of white cannot be produced by any single unitary physical stimulation. It requires the simultaneous action of light from more than one region of the spectrum. This seems to me a fundamental consideration, because if a simple sensation like white can be produced only by a heterogeneous stimulation, it is possible for a simple sensation like yellow to be the result of a heterogeneous stimulation. The sensation of white can be produced by stimulation by light from the whole of the spectrum, or from three or from two selected regions. There is no fixed standard of white. A white surface is one that reflects all visible wave-lengths well and equally. In order to define a 'white' light a standard is taken of the radiation of a perfect radiator at $4,800^{\circ} \mathrm{K}$. or other specified temperature. When a white sensation is produced by light from two regions of the spectrum, the separate sensations produced by these radiations are said to be complementary.

Black sensation cannot be produced by any combination of radiations. It is always the result of a relative deficiency of stimulation. A black surface is one that does not reflect any visible wave-length to an appreciable extent. To produce a black effect with spectral lights a brighter light must shine alongside them. Thus, a red produced by wave-length of about $6500 \mathrm{~A}$. looks brown when a bright yellow produced by wave-length of about $5900 \mathrm{~A}$. shines alongside of it.

The transition between white and black through grey depends upon the relative amount of illumination. There must, however, be the right mixture of wave-lengths, otherwise the grey will be tinted with the colour sensation produced by those wavelengths which are in excess.

We are now in a position to consider the phenomenon of yellow. Yellow is a unitary 
sensation which can be produced by a single group of wave-lengths or by two groups, one each on the 'red' and 'green' sides of the 'yellow' region. If we are to believe that three types of sensory mechanism are sufficient to account for colour vision, one of the four colours red, yellow, green and blue must be due to a stimulation of at least two of the other ones. For several reasons, yellow has been chosen as the heterogeneous one.

To my mind there is no more difficulty in considering yellow as due to stimulation of two types of receptors than to consider white as due to stimulation of more than one type. Experimental evidence supports this view. Macdougall, Rochat, and others have shown that a 'red' stimulus to one eye and a 'green' to the other will give a sensation of yellow. This result is obtained even with lights from the spectrum. The fact has been demonstrated by Hecht, but his method is not such a satisfactory proof as that obtained by other methods, for example, a 'red' glass over one eye and a 'green' one over the other, or two definite wave-lengths of the spectrum each presented to one eye.

Central summation of this type shows that the sensation is built up in the nervous system beyond the optic chiasma, as neither eye need be stimulated by the 'yellow' of the spectrum. The red and green sensations are lost, but their disappearance cannot be due to processes in the layers of the retina. As Macdougall points out, the alternative suggested by Hering that his four-dimensional system is cerebral rather than retinal deprives his hypothesis of its special value as a theory of colour vision. Hering's theory then becomes part of a general problem of how afferent stimuli are combined to produce perceptions, which is too complex a matter to be discussed here.

As the unitary sensations yellow, white and black can be built up from stimuli associated with other sensations, it is possible to reduce the number of data for colour perception to three.

The object of the above discussion is to show that there is no real objection to the trichromatic explanation of colour vision proposed by Thomas Young.

\section{Defective Colour Vision}

Abnormal colour vision may be congenital or acquired. It is not my intention to discuss certain defects in colour vision due to disease, for example, tobacco amblyopia.

Defective colour vision is a condition in which the persons affected make mistakes in matching colours. Any explanation of the nature of colour vision must be able to explain how certain colours are mistaken. The usual form of defective colour vision is congenital, and does not alter during life.
This is what is generally understood when speaking of defective colour vision. The defect seems to consist in a decrease in the ability to distinguish 'red' from 'green', and the subjects distinguish fewer colours than the normal (euchromat); hence they may be spoken of as hypochromats. It is very difficult to compare the sensations of such cases with those of a normal person, but they are frequently described as having blue-yellow vision. Another way of expressing the fact is to say that in the spectrum they distinguish blue from not blue, whereas the normal person subdivides the not blue into red and green. As 'yellow' occupies the region between 'red' and 'green', the defect is most noticeable in the 'yellow' region of the spectrum, especially in the milder degrees of the defect.

Part of the evidence for these statements is that analysis of the mistakes made by hypochromats are all explained by a failure to distinguish red from green. Further evidence is furnished by observations on colour discrimination.

By measuring the difference in wave-length necessary to cause a difference in colour, it is found that normal people have two main maxima of discrimination where a difference in colour is recognised for a minimal change in wave-length. These maxima of discrimination probably indicate where there is a most rapid change in the ratio of stimulation of two different types of receptor organs. The hypochromat shows only one maximum of discrimination, thus suggesting that he has only two types of receptor organs.

In extreme degrees of this defect, the whole range of colours can be reproduced for these people by fusion of light from two regions of the spectrum.

The normal maxima are in the 'yellow' and 'blue-green' of the spectrum, whilst the hypochromat has only one maximum, that in the 'blue-green'. It appears as if the distinction on each side of the yellow had been diminished or lost: hence the failure to distinguish 'red' from 'green', and the whole not blue portion of the spectrum appears more or less of one colour. The bearing of this on any theory of vision is that we must be able to explain how the distinction between red and green can disappear, yet without marked decrease in the visibility of any portion of the spectrum. The threshold for light is not necessarily altered, and it is possible for hypochromats to see clearly through a filter which allows only the red end of the spectrum to pass through. In fact, a hypochromat who cannot see red geraniums amongst the green leaves can distinguish the flowers as light objects against a dark background when looking through a red glass filter. 


\section{Theories of Colour Viston}

In order to explain the phenomena of colour vision, it seems that it is necessary to have three groups of nerve fibres passing to the brain-one group giving rise to sensation $\mathrm{A}$, a second to sensation $\mathrm{B}$, and a third to sensation $\mathrm{C}$. We must discover what wave-lengths stimulate A, B and $\mathrm{C}$ respectively, what sensations are produced by stimulation of one of these alone, and what is the effect of stimulating more than one of these, either to the same degree for each or to different ratios of response. Stimulation of the receptors may correspond to definite wave-length groups, but there may be a certain amount of rearrangement in the retinal synapses. It does not seem probable that the number of types of receptors or groups of nerve fibres can be reduced below three, if frequency of the impulses is to be related to intensity of stimulation, and if only one kind of impulse can pass up each fibre. It is like the solution of simultaneous equations: the number of equations must be at least equal to the number of unknowns to be found. That seems to be the essence of the trichromatic hypothesis as suggested by Young.

Helmholtz introduced the view that the differentiation is due to the presence of three photoactive substances which are acted on by the long, medium and short wave-lengths of the visible spectrum respectively. The range of radiation which affects these three substances overlaps so that, for example, some rays affect all three of these substances. Up to the present, there is no definite evidence for the presence of three photoactive substances; only one photo-active substance, rhodopsin or visual purple, has been found. Apart from this fact, the view of three photochemical substances such as postulated by Helmholtz does not agree with the experimental evidence. For example, in order to explain hypochromatism, it is not assumed that one photo-active substance is absent, but that the range of activity has shifted so that the one substance is activated by the range which was formerly active on the two separate substances. It does not seem likely that such a chemical transformation would occur.

Another suggestion is that put forward by Schultz (1866), namely, that there is one photochemical substance but different coloured filters to distinguish the various regions of the visible spectrum. Such filters have been found in amphibians, reptiles, birds and marsupials, but have not been found in other mammals. The coloured filters in the birds' retinæ would explain the type of colour vision found in man. For example, by reducing the intensity of red pigment in the red filters the various degrees of hypochromatic vision would be produced, but in a single human eye examined by me no such filters could be seen.

My own work leads me to suppose that the types of receptors which are stimulated by visible radiation are as follow :-

The first type of receptor is one which is stimulated by all parts of the visible spectrum and gives rise to a sensation of violet when stimulated strongly by itself. It is with some hesitation that one states that violet is due to stimulation of a single receptor, as psychologically it suggests a mixture of blue with a little red. If violet is the sensation corresponding to stimulation of one type of receptor, we must regard the unitary sensation of blue as due to stimulation of the receptors for green and violet. It may be that blue is the sensation due to stimulation of the single receptor, and that violet is the result of stimulation of the receptors which give rise to blue and to red sensations. This matter must be left in abeyance, but the use of the term 'violet receptor' is to be understood to mean either the receptor for violet or blue. Owing to the fact that fatigue to 'red' causes violet to appear more blue, Wright believes that the single receptor gives rise to a sensation of blue.

The evidence for the first part of the above statement is the same as that which caused Hering to speak of a white-black substance and von Kries to describe a bluish-white sensation as due to stimulation of the receptors for achromatic scotopic vision: these usually being regarded as the rods.

The evidence for the second part of the above statement is first of all that a narrow beam of any wave-length when shining slightly eccentrically gives rise to a violet sensation. This has been called secondary excitation, implying that the sensation is due to stimulation of receptors by nerve impulses passing along fibres of the optic nerve. It is unlikely that such stimulation would occur, and if so, why should the sensation produced be violet? On the whole, it seems simpler to interpret it as stimulation of rods by any wavelength. Furthermore, diseases involving the rods lead to night blindness or raising the threshold of achromatic scotopic vision. If this threshold is sufficiently raised, then there is loss of vision for violet, so that the distinction between green and blue is lost. This defect is a true violet blindness, because it is accompanied by a raised threshold for the short wave-length end of the spectrum. Finally, adaptation to light conditions is accompanied by a special raising of the threshold to the short wave-length end of the spectrum. Therefore, although the point is not proved, there is much evidence in favour of violet vision being a function of the rods. 
The second type of receptor is one which is concerned with the not blue aspect of vision of the hypochromat. These may be cones, of which there need be only one variety for the hypochromat.

The third type of receptor would be functional in normal vision, and it seems as if this second variety of cone were one that distinguishes red from not red, and according to the activity of this variety the stages between normal vision and complete red-green confusion can be bridged.

Therefore, normal vision may be due to a receptor which gives rise to a red sensation, one which gives rise to a blue sensation and one which gives rise to a not blue, not red sensation which, of course, corresponds to green sensation. The actual wave-lengths of radiation that stimulate the several receptors are not known. The real difference between various hypotheses is the extent and region of the spectrum which stimulates the end-organs.

In the Young-Helmholtz hypothesis the type of receptor responsible for the sensation of red is stimulated by almost the whole of the spectrum, but most strongly by the long wave-length end. The receptors for green are stimulated by almost the whole spectrum, but most strongly by the midregion; and those for blue are stimulated by a large extent of the spectrum, but most strongly by the short wave-length end.

'Red' light of longer wave-length than $6200 \mathrm{~A}$. is supposed to stimulate the red receptor only, whilst shorter wave-lengths will stimulate the red receptor to decreasing degrees, but the other receptors to increasing extent, hence the change of colour with wave-length.

\section{Sources of Cheap Electric Power* By Prof. Francis G. Baily}

$\mathrm{T}$ HE general idea of the scheme of production of electric energy proposed takes as its basis the complete linking up of all parts of Great Britain by the Grid, and the subsidiary lines fed from it or from the stations directly. All stations are connected to the Grid, and as well as supplying their local consumers, put the additional power into the Grid as required. This is the well-known main function of the Grid. It is here submitted that this leads to a different scheme of generation from that now followed, and that sources of cheap power are rendered available that previously could not be utilised economically.

The questions to be considered are : (1) The proportion of consumers who are within economic distance of a pit-head station. (2) The quantity of very cheap coal that is available. (3) The relative advantages of widely spaced large stations and more numerous small stations. (4) The opportunity offered by the Grid to bring into economical use pit-head stations at small isolated mines, power from factories using industrial steam, power from coke-oven and blast-furnace gas and hydroelectric stations. (5) The cost of transmission of electric power as compared with the carriage of the equivalent coal by rail or ship. (6) The effect of a substantial reduction in the cost of generation on the cost of distribution, and the selling price of electric energy.

The first question to be considered is whether pit-head production will so much limit the position of the sources of supply as to involve a great distance of transmission to a large part of the population.

- From the presidential address before Section $G$ (Engineering) of the British Association, delivered at Aberdeen on September 6.
If a distance of forty miles be regarded as still in the neighbourhood of the coalfields, a map of the coalfields shows that most of Great Britain is within this distance. A line across Scotland from Montrose to Arrochar on Loch Long is the northern boundary, and a line from Hull to Bournemouth, and up to Taunton in Somerset, marks the southern and eastern limits. A small part of Wales is also outside. Two-thirds of the population live in the area, and if London be omitted as a special case, only one-fifth of the rest are outside. There is also a probable coalfield in Lincolnshire, which if it materialises will bring in a good part of this fifth.

To a large extent, the population has gathered round the coal pits, and there are practically no large towns, except seaports, that do not lie within easy reach. A scheme depending on nearness to coal pits will have a large field for its operations. and it will in no way act prejudicially on parts which it may not be able to benefit.

It is proposed to use the lowest grade and waste coal, and the proportion required may be up to 10 per cent of the total coal raised. If the outputs of the different areas be examined, it is found that this proportion will in all cases be adequate for the population of the area. In some areasDurham, South Wales and part of Yorkshirewhere there is much less waste coal, the quantity of coal raised is so large that not more than 2 per cent will be required, which is easily provided from waste.

\section{WASTE COAL}

The term 'waste coal' will here be used to include all coal in the seam that is not at present sold, but 